\title{
The Effect of Different Laser Irradiation on Cyclophosphamide-Induced Leucopenia in Rats
}

\author{
Jizhong Zhao, Ke Cheng, Haiping Deng, Ling Zhao, Lanlan Liu, Menghu Guo, \\ Haimeng Zhang, and Xueyong Shen
}

Acupuncture \& Tuina College, Shanghai University of Traditional Chinese Medicine, Shanghai 201203, China

Correspondence should be addressed to Ling Zhao; zl2@shutcm.edu.cn

Received 9 December 2013; Revised 20 March 2014; Accepted 28 April 2014; Published 14 May 2014

Academic Editor: Lixing Lao

Copyright (c) 2014 Jizhong Zhao et al. This is an open access article distributed under the Creative Commons Attribution License, which permits unrestricted use, distribution, and reproduction in any medium, provided the original work is properly cited.

\begin{abstract}
Objective. To assess the effect of different lasers on cyclophosphamide- (CTX-) induced leucopenia in rats. Methods. 11 rats were normal control and 55 rats were injected with a dose of $80 \mathrm{mg} / \mathrm{kg}$ CTX for the first time and $40 \mathrm{mg} / \mathrm{kg}$ on the 6th and the 11th days to establish a leucopenia model. Rats of the irradiation groups received a 5-minute laser irradiation with either single $10.6 \mu \mathrm{m}$ or $650 \mathrm{~nm}$ laser or alternatively $10.6 \mu \mathrm{m}-650 \mathrm{~nm}$ laser irradiation, besides a sham treatment on acupoint Dazhui (DU 14) and acupoint Zusanli (ST 36) of both sides, 8 times for 16 days. Normal and model control group received no treatment. Results. On day 16 after the first CTX injection, the WBC counts from all the laser irradiation groups were significantly higher than those from the model control and the sham group $(P<0.05)$, while there were no significant differences compared with the normal control $(P>0.05)$. The TI of $10.6 \mu \mathrm{m}-650 \mathrm{~nm}$ laser irradiation group was significantly higher than that of the model control group $(P<0.05)$. Conclusions. The single and combined $10.6 \mu \mathrm{m}$ and $650 \mathrm{~nm}$ laser irradiation on ST36 and DU14 accelerated the recovery of the WBC count in the rats with leucopenia.
\end{abstract}

\section{Introduction}

Leucopenia, a disease with a white blood cell (WBC) number consistently lower than $4.0 \times 10^{9} / \mathrm{L}$ in the peripheral blood, can be classified into primary and secondary leucopenia. The main secondary cause of leucopenia is bone marrow suppression that resulted from antitumor drugs. Cancer chemotherapy drugs have long been considered immune suppressive [1]. Bone marrow suppression can severely influence the effect of chemotherapy as well as quality of life after chemotherapy. As a main approach for antitumor therapy, chemotherapy can improve the pathological state of the patients. However, its side effects, such as bone marrow suppression, also bring harm to the patients' physical, mental, and economical states. Clinical studies showed that acupuncture and moxibustion associated with relief of side effects and increase of leukocyte in patients with leucopenia induced by chemotherapy. However, the evidence was of low quality [2-5]. Animal studies found that electroacupuncture and acupoint injection showed benefit on bone marrow suppression after chemotherapy, through protection of hematopoietic function, improvement of WBC counts in the peripheral blood, and enhancement of immune function $[6,7]$. However, needling acupuncture might result in some degrees of discomfort, such as pain, while moxibustion produces smog, which could be irritant and harmful to the health of both the practitioners and patients [8-10]. These factors limited the application of traditional acupuncture in the clinical use. Laser acupuncture/moxibustion is a good substitute of traditional acupuncture/moxibustion and has been researched for more than 20 years [11]. Laser acupuncture/moxibustion causes no skin penetration and smoke and is easier to practice and regulate. Our previous research found that the $\mathrm{CO}_{2}$ laser with the wavelength of $10.6 \mu \mathrm{m}$ and semiconductor laser with the wavelength of $650 \mathrm{~nm}$ have shown some benefit for pituitrin-induced bradycardia and knee osteoarthritis $[12,13]$. The purpose of this study was to explore the effect and the mechanism of acupoint irradiation with the single $10.6 \mu \mathrm{m}$ 
$\mathrm{CO}_{2}$ laser, the single $650 \mathrm{~nm}$ semiconductor laser, and the combination of the two lasers in cyclophosphamide- (CTX-) induced leucopenia.

\section{Materials}

2.1. Animals. A total of 66 healthy adult male SD rats were supplied by Animal Experiment Center of Shanghai Traditional Chinese Medicine University, with body weight of 200 $\pm 20 \mathrm{~g}$ and normal white blood cell (WBC) count. The license number for animal manufacture was SCXK (HU) 2009-0018, bought from Shanghai Xipuer-Bikai Lab Animal Co., Ltd. The license number for the use of experimental facilities in Shanghai University of Traditional Chinese Medicine was SCXK (HU) 2009-0069.

2.2. Reagents. The CTX $(0.2 \mathrm{~g})$ used to induce leucopenia was bought from Jiangsu Hengrui Pharmaceutical Incorporated Company. The manufacturing lot number for it was H32020857.

2.3. Apparatus. The $\mathrm{CO}_{2}$ laser acupuncture apparatus (SX10$\mathrm{C1}$ ) and the $650 \mathrm{~nm}$ semiconductor laser acupuncture apparatus were produced by Shanghai Wanqi Photoelectric Technology Co., Ltd. The wavelength of the $\mathrm{CO}_{2}$ laser was $10.6 \mu \mathrm{m}$ and its output power was $55 \pm 5 \mathrm{mw}$. The wavelength of the semiconductor laser was $650 \mathrm{~nm}$ and its output power was $36 \mathrm{mw}$ with a diameter of light spot of $1 \mathrm{~mm}$ [12]. ANI fullautomatic animal blood cell analyzer (XFA6030) was bought from Nanjing Pulang Medical Equipment Co., Ltd.

\section{Methods}

3.1. Establishment of Rat Leucopenia Model. All animals were raised and bred in the same standard environment, with the room temperature of $(24 \pm 1)^{\circ} \mathrm{C}$ and humidity of $50-70 \%$. An improved method to establish animal model for leucopenia was used $[14,15]$. The rats were weighed and CTX was used for intraperitoneal injection in a body weight-dependent dose manner. A total of $80 \mathrm{mg} / \mathrm{kg}$ of CTX was injected for the first time and $40 \mathrm{mg} / \mathrm{kg}$ on the 6 th and the 11th days.

3.2. Selection and Location of Acupoints. Acupoint Dazhui (DU 14) and acupoint Zusanli (ST 36) of both sides were selected on the basis of TCM theory as well as the previous clinical studies [16]. Acupoints were located referring to standards described in "Experimental Acupuncture Science" [17]. We located Zusanli at posterolateral knee joint, about $5 \mathrm{~mm}$ underneath the fibulae capitulum, and Dazhui between the spinous process of the 7 th cervical vertebra and the 1st thoracic vertebra, in the middle of the back [17].

3.3. Animal Grouping. Rats were numbered according to their body weight. A total of 66 rats were randomly allocated into six groups with a random number produced by SPSS18.0. These groups included normal control group, model control group, sham laser irradiation group, $10.6 \mu \mathrm{m} \mathrm{CO}$ laser irradiation group, $650 \mathrm{~nm}$ semiconductor laser irradiation group, and combined $10.6 \mu \mathrm{m}-650 \mathrm{~nm}$ laser irradiation group. With the exception of normal control group, the rats in the other five groups were induced by leucopenia with CTX. After inducing leucopenia, the 3 true laser groups were irradiated on the acupoint Dazhui (DU 14) and bilateral acupoint Zusanli (ST 36) with $10.6 \mu \mathrm{m} \mathrm{CO}_{2}$ laser, $650 \mathrm{~nm}$ semiconductor laser, and combined $10.6 \mu \mathrm{m}-650 \mathrm{~nm}$ laser, respectively. Each acupoint received irradiation for 5 minutes (min) every other day, with a total of 8 sessions of irradiation. The sham laser irradiation group was treated the same way as true laser groups, but without laser output.

\subsection{Sample Collection and Detection}

3.4.1. Detection of WBC Counts in the Peripheral Blood Collected from Tail End of the Rats. Rat tail was cut and blood was collected into tubes with anticoagulant one day before as well as 1, 4, 15, and 16 days after the CTX injection. Animal blood cell analyzer was used to count the blood cell numbers. WBC counts were also recorded.

3.4.2. Calculation of Thymus Index (TI). Thymus gland was excised from each rat on the 16th day after the first injection of CTX, washed with physiological saline, and weighed using a balance with 0.0001 accuracy. The TI was calculated with the formula TI $=$ (index of thymus weight/body weight $) \times 10$.

3.5. Statistical Analysis. The SPSS18.0 software was used for statistical analysis. The outcome data was expressed as mean \pm standard deviation (mean \pm SD) when the data accords with normal distribution. One-way ANOVA was used for comparison between groups and ANOVA for repeated measurement method was used in comparison within groups. The TI was analyzed with one-way ANOVA and the differences were considered statistically significant when the $P$ was less than 0.05 .

\section{Results}

4.1. Establishment of Rat Leucopenia Model. Before the first CTX injection, WBC counts of the rats in all groups were normal showing no statistically significant differences $(P>$ 0.05). After CTX injection, the WBC counts in the other 5 groups significantly decreased compared with the normal control group $(P<0.05)$, while there were no statistically significant differences among the 5 groups $(P>0.05)$. These results indicated that we successfully established the leucopenia model in rat (Table 1). There was one rat that died after CTX injection in each of the model control, sham irradiation, $10.6 \mu \mathrm{m}$ laser irradiation group, and $10.6 \mu \mathrm{m}-$ $650 \mathrm{~nm}$ irradiation group.

4.2. Changes of WBC Counts after Laser Irradiation. Results showed that WBC counts decreased after the first CTX injection and then rose at day 16. On the first and fourth days, WBC counts were significantly lower in all the other 5 groups compared to that of the normal control group $(P<$ 0.01). Sixteen days after first CTX injection, the WBC counts 
TABLE 1: WBC counts in rat peripheral blood (mean $\left.\pm \mathrm{SD}, \times 10^{9}\right)$.

\begin{tabular}{|c|c|c|c|c|c|}
\hline \multirow{2}{*}{ Group name } & \multirow{2}{*}{ Animal number } & \multirow{2}{*}{ Before the first CTX injection } & \multicolumn{3}{|c|}{ After the first CTX injection } \\
\hline & & & $1 \mathrm{~d}$ & $4 \mathrm{~d}$ & $16 \mathrm{~d}$ \\
\hline Normal control & 11 & $11.70 \pm 4.36$ & $7.72 \pm 2.85$ & $9.47 \pm 3.9$ & $11.17 \pm 1.48$ \\
\hline Model control & 10 & $13.87 \pm 3.12^{1}$ & $2.50 \pm 0.69^{2}$ & $2.48 \pm 2.01^{2}$ & $6.24 \pm 3.37^{2}$ \\
\hline Sham irradiation & 10 & $14.19 \pm 2.94^{1}$ & $1.26 \pm 0.61^{2}$ & $1.20 \pm 0.54^{2}$ & $7.33 \pm 4.17^{2}$ \\
\hline $10.6 \mu \mathrm{m}-650 \mathrm{~nm}$ & 10 & $11.25 \pm 1.77^{1}$ & $0.76 \pm 0.62^{2}$ & $1.35 \pm 1.34^{2}$ & $10.01 \pm 4.17^{1,3,4}$ \\
\hline $650 \mathrm{~nm}$ & 11 & $11.83 \pm 2.06^{1}$ & $1.88 \pm 0.61^{2}$ & $2.42 \pm 0.95^{2}$ & $10.11 \pm 3.11^{1,3,4}$ \\
\hline $10.6 \mu \mathrm{m}$ & 10 & $11.66 \pm 2.17^{1}$ & $1.26 \pm 0.47^{2}$ & $1.68 \pm 1.10^{2}$ & $11.89 \pm 5.56^{1,3,4}$ \\
\hline
\end{tabular}

Compared with normal control: ${ }^{1} P>0.05,{ }^{2} P<0.05$; compared with model control: ${ }^{3} P<0.05$; compared with sham irradiation: ${ }^{4} P<0.05$.

TABLE 2: The TI of each group 16 days after model establishment.

\begin{tabular}{lccr}
\hline Group name & Animal numbers & Weight $(\mathrm{g})$ & TI \\
\hline Normal control & 11 & $307.36 \pm 20.92$ & $1.7637 \pm 0.1257$ \\
Model control & 10 & $240 \pm 12.27$ & $0.6327 \pm 0.0610^{1}$ \\
Sham irradiation & 10 & $232.5 \pm 17.46$ & $0.8773 \pm 0.0765^{1}$ \\
$10.6 \mu \mathrm{m}-650 \mathrm{~nm}$ & 10 & $244.5 \pm 19.03$ & $1.1174 \pm 0.2950^{2}$ \\
$650 \mathrm{~nm}$ & 11 & $243.73 \pm 19.92$ & $0.9361 \pm 0.0961^{1}$ \\
$10.6 \mu \mathrm{m}$ & 10 & $242.1 \pm 19.90$ & $0.8668 \pm 0.0902^{1}$ \\
\hline
\end{tabular}

Compared with normal control group: ${ }^{1} P<0.05$; compared with model control group: ${ }^{2} P<0.05$.

returned to normal level in the 3 true laser irradiation groups with no statistically significant difference compared to that in the normal control group $(P>0.05)$. WBC counts were still lower in the model control and sham irradiation groups as compared to that in the normal control group $(P<0.05)$ (Table 1).

Sixteen days after the first CTX injection, the WBC counts were statistically significantly higher in each group treated with laser irradiation as compared to that in the model control group $(P<0.05)$ and sham irradiation group $(P<$ $0.05)$. The WBC counts in the sham irradiation and model control groups showed no statistically significant difference at any of the time points $(P>0.05)$.

4.3. Thymus Index. Sixteen days after the first CTX injection, the TI in each group with laser irradiation was significantly lower than that of the normal control group $(P<0.01)$, while the TI in the $10.6 \mu \mathrm{m}-650 \mathrm{~nm}$ laser group was statistically significantly higher than that of the model control group $(P<$ 0.05) (Table 2).

\section{Discussion}

Our result indicated that $\mathrm{WBC}$ counts in groups with irradiation of $10.6 \mu \mathrm{m} \mathrm{CO}$ laser, $650 \mathrm{~nm}$ semiconductor laser, and $10.6 \mu \mathrm{m}-650 \mathrm{~nm}$ laser recovered more quickly than those in the sham irradiation and model control groups. Since there was no difference between the WBC counts in the sham irradiation and model control groups, the effect of the factors other than laser acupuncture stimulation on experimental results, such as the fixation of rats, might be excluded. This finding was similar to our previous pilot study, in which we found similar therapeutic effect of $10.6 \mu \mathrm{m} \mathrm{CO} 2$ laser and
$650 \mathrm{~nm}$ semiconductor laser for the CTX-induced leucopenia in rat.

Thymus gland reflects the state of the immune function, which might be suppressed by chemotherapy. Results showed that only the combination of $10.6 \mu \mathrm{m}-650 \mathrm{~nm}$ laser irradiation improved the TI of the leucopenia model rats, while neither $10.6 \mu \mathrm{m}$ laser nor $650 \mathrm{~nm}$ laser alone improved the TI, indicating that the combination of both lasers is necessary to improve the immune function, which is in accordance with the previous research findings [18].

In the view of traditional Chinese medicine, leucopenia belongs to the category of consumptive disease and blood deficiency. The chemotherapy drugs are normally working well to treat cancerous cell; however, their toxicity is harmful to Qi and blood, as well as the function of Zang and Fu organs. Stomach is a reservoir of food and drink as well as a source of Qi and blood. Zusanli (ST 36), located on the stomach meridian, can regulate and tonify the spleen and stomach Qi, thus regulating and facilitating Qi and blood. Dazhui (Du 14), the crossing point of all Yang meridians on the Du meridian, can invigorate Yang Qi and blood. Both ST 36 and DU 14 are the most commonly used acupoints in treating leucopenia as reported [16].

A general method used for model establishment with the CTX is to inject animals only one time, lasting for 1 to 5 days according to the dose. We have used this method in our previous study and established a model for rat leucopenia, which lasted for 10 days [18]. After iterative trial and error, we finally chose the method of multiple injection of CTX at a low dose in order to establish the model for rat leucopenia. With this method, the established model lasted for about 16 days, which was considered as a relatively steady model that replicated leucopenia caused by clinical chemotherapy and was convenient for observation of curative effects. 
The $\mathrm{CO}_{2}$ laser is a far-infrared light with the wavelength of $10.6 \mu \mathrm{m}$, which can be absorbed by epidermis within $0.2 \mathrm{~mm}$ and can bring about a rapid, obvious, and abiding heat effect [19]. The $650 \mathrm{~nm}$ semiconductor laser can reach the deep epidermis, stimulate several sensors on the nerve ending at the acupoint, and result in effects similar to those of acupuncture. Our previous study on animals indicated that $10.6 \mu \mathrm{m}-650 \mathrm{~nm}$ laser irradiation on acupoint Neiguan had effect on pituitrin-induced bradycardia in rabbit, with $\mathrm{CO}_{2}$ laser playing the main role in this action [12].

In conclusion, irradiation with the $10.6 \mu \mathrm{m}$ laser, $650 \mathrm{~nm}$ laser, and combined $10.6 \mu \mathrm{m}-650 \mathrm{~nm}$ laser on certain acupoints boosted the recovery of WBC counts in the peripheral blood and improved the hematopoietic function of the rats with leucopenia. The combination of both lasers might be necessary to improve the immune function. This approach and its mechanism of action need further investigations.

\section{Conflict of Interests}

The authors declare that there is no conflict of interests regarding the publication of this paper.

\section{Authors' Contribution}

Jizhong Zhao, Ke Cheng, and Ling Zhao contributed equally to this study and should be considered as cofirst authors.

\section{Acknowledgments}

This study was supported by 973 Program of China (2009CB522901), Shanghai Science and Technology Developing Foundation (11ZR1436700, 11DZ1973300), Projects of NNSF of China $(81320108028,81202648)$, the Key Program of the SATCM of China (ZYSNXD-CC-ZDXK-07), and 2014 Innovation Program of the Shanghai Municipal First-Class Field of Traditional Chinese Medicine of Shanghai.

\section{References}

[1] G. Chen and L. A. Emens, "Chemoimmunotherapy: reengineering tumor immunity," Cancer Immunology, Immunotherapy, vol. 62, no. 2, pp. 203-216, 2013.

[2] X.-X. Zhao, M. Lu, X. Zhu et al., "Multi-central clinical evaluation of ginger-partitioned moxibustion for treatment of luekopenia induced by chemotherapy," Zhongguo Zhen Jiu, vol. 27, no. 10, pp. 715-720, 2007 (Chinese).

[3] Y.-F. Han, Z. Gong, L.-Q. Huang, X. Xia, and W.-J. Zhao, "Clinical study on acupuncture for leukopenia induced by chemotherapy," Zhongguo Zhen Jiu, vol. 30, no. 10, pp. 802-805, 2010.

[4] W. Lu, U. A. Matulonis, A. Doherty-Gilman et al., "Acupuncture for chemotherapy-induced neutropenia in patients with gynecologic malignancies: a pilot randomized, sham-controlled clinical trial," Journal of Alternative and Complementary Medicine, vol. 15, no. 7, pp. 745-753, 2009.

[5] W. Lu, D. Hu, E. Dean-Clower et al., "Acupuncture for chemotherapy-induced leukopenia: exploratory meta-analysis of randomized controlled trials," Journal of the Society for Integrative Oncology, vol. 5, no. 1, pp. 1-10, 2007.

[6] X. Yin, D. Yin, X. Liu, and X. Ding, “Treatment of 104 cases of chemotherapy-induced leukopenia by injection of drugs into Zusanli," Journal of Traditional Chinese Medicine, vol. 21, no. 1, pp. 27-28, 2001.

[7] H.-J. Mao, H.-H. Wu, L.-L. Bu, Y.-B. Zhou, J. Sun, and P.-L. Sun, "Relationship between electroacupuncture-induced regulatory effect on leukocytes and the caliber of splenic sinusoid basal lamina eyehole on rabbits," Zhen Ci Yan Jiu, vol. 33, no. 5, pp. 291-295, 2008 (Chinese).

[8] H. Sakagami, H. Matsumoto, K. Satoh et al., "Cytotoxicity and radical modulating activity of Moxa smoke," In Vivo, vol. 19, no. 2, pp. 391-397, 2005.

[9] J. Wheeler, B. Coppock, and C. Chen, "Does the burning of moxa (Artemisia vulgaris) in traditional Chinese medicine constitute a health hazard?" Acupuncture in Medicine, vol. 27, no. 1, pp. 16-20, 2009.

[10] N. Hitosugi, R. Ohno, J. Hatsukari et al., "Diverse biological activities of Moxa extract and smoke," In Vivo, vol. 15, no. 3, pp. 249-254, 2001.

[11] P. Whittaker, "Laser acupuncture: past, present, and future," Lasers in Medical Science, vol. 19, no. 2, pp. 69-80, 2004.

[12] L. Zhao, X. Y. Shen, J. P. Gao et al., "Effect of different LLLT on pituitrin induced bradycardia in the rabbit," Lasers in Medical Science, vol. 21, pp. 61-66, 2006.

[13] X. Shen, L. Zhao, G. Ding et al., "Effect of combined laser acupuncture on knee osteoarthritis: a pilot study," Lasers in Medical Science, vol. 24, no. 2, pp. 129-136, 2009.

[14] A. Jacolot, C. Judel, M. C. Chaumais et al., "Animal model methodology: immunocompetent or leucopenic rats, which is the best? Results from a model of experimental pneumonia due to derepressed cephalosporinase-producing Enterobacter cloacae," Chemotherapy, vol. 58, pp. 129-133, 2012.

[15] X.-H. Huyan, Y.-P. Lin, T. Gao, R.-Y. Chen, and Y.-M. Fan, "Immunosuppressive effect of cyclophosphamide on white blood cells and lymphocyte subpopulations from peripheral blood of Balb/c mice," International Immunopharmacology, vol. 11, no. 9, pp. 1293-1297, 2011.

[16] Q. F. Huang, X. J. Cui, and S. Li, "Review of Leucopenia treated by acupuncture and moxibustion from 1949 to 2004," Jounnal of Acupuncture and Tuina Science, vol. 4, no. 3, pp. 129-131, 2006.

[17] L. Zhongren, Experimental Acupuncture Science, vol. 242, Chinese Publishing House of Traditional Chinese Medicine and Pharmacology, Beijing, China, 2007.

[18] L. Zhao, H. Deng, X. Shen, and H. Zhang, "Effect of different laser acupuncture on rats with Leucopenia," in Proceedings of the Conference of the Society for Acupuncture Research, Chapel hill, Calif, USA, 2010.

[19] Z. Li and Z. Zhang, "The mechanism on laser acupuncture and moxibustion," Acta Laser Biology Sinica, vol. 14, pp. 8-11, 2005. 


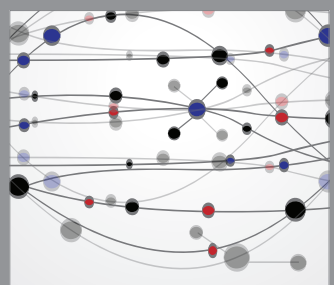

The Scientific World Journal
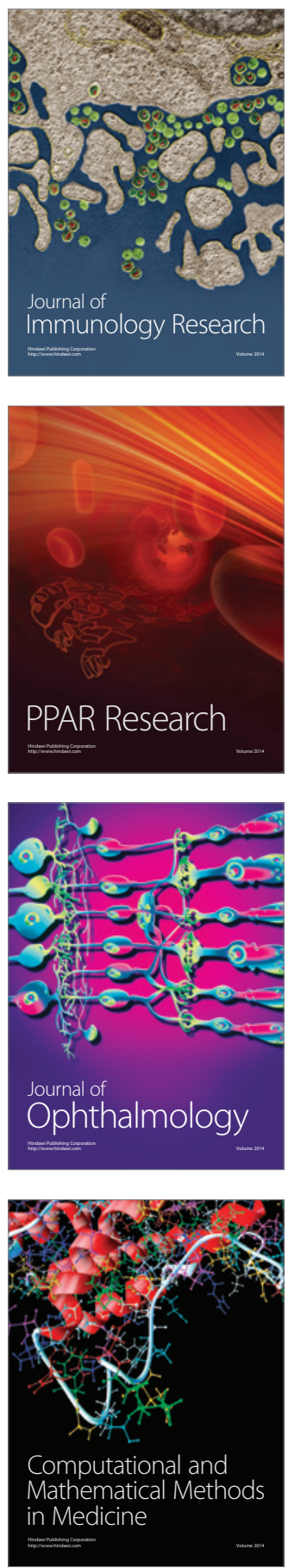

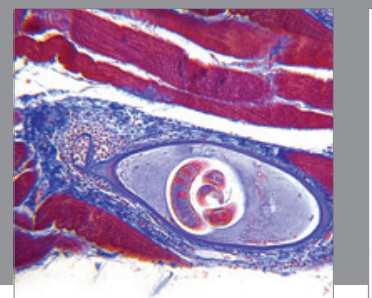

Gastroenterology

Research and Practice
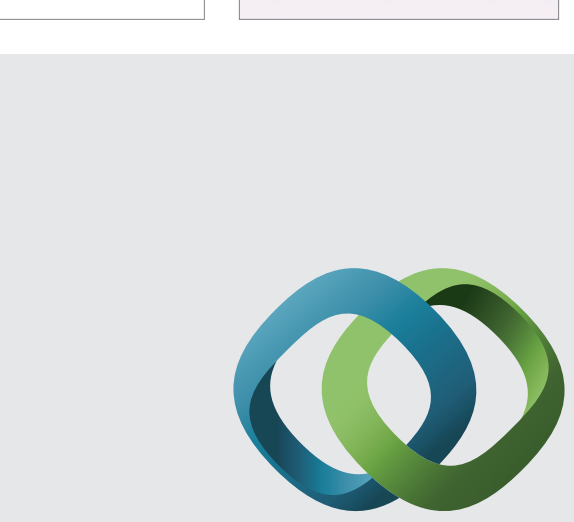

\section{Hindawi}

Submit your manuscripts at

http://www.hindawi.com
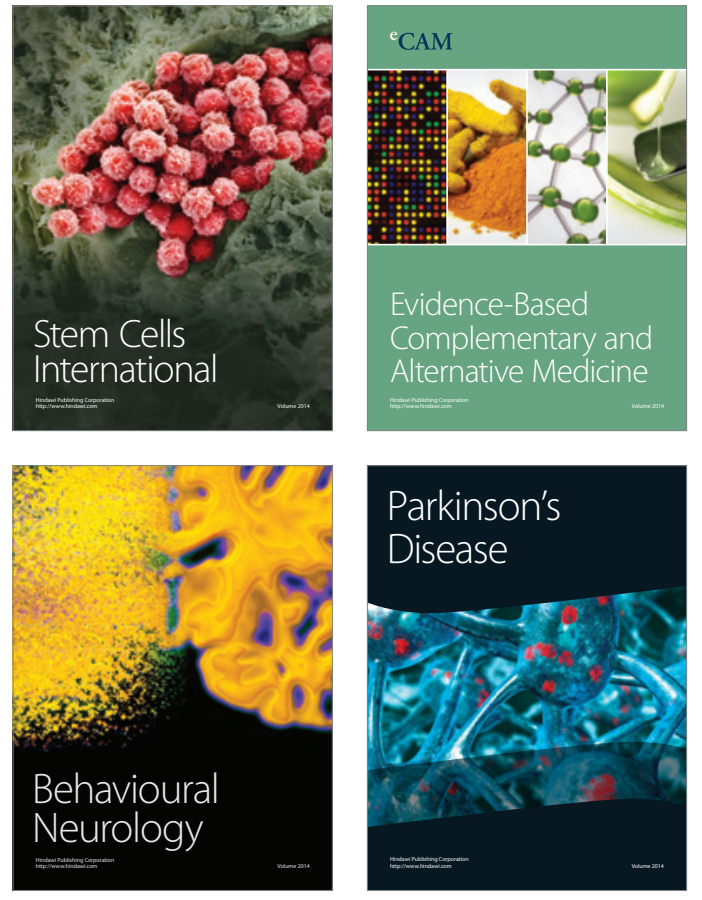
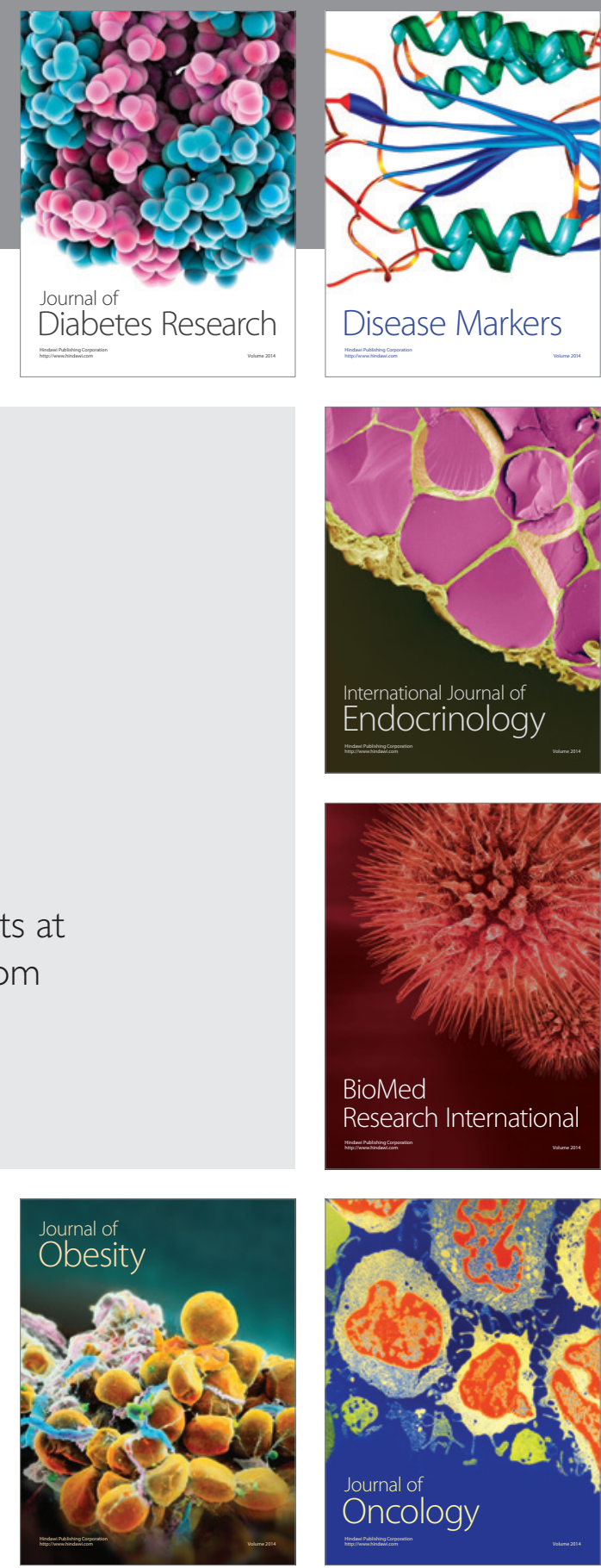

Disease Markers
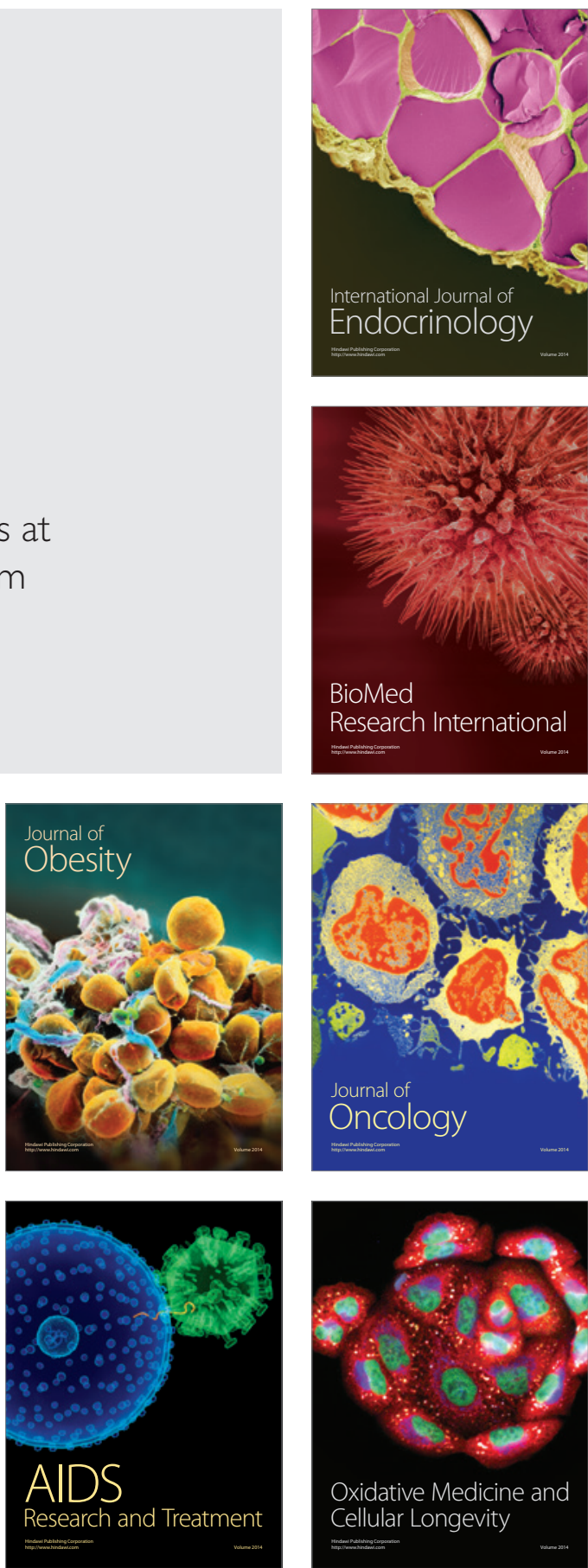